\title{
Molecular interplay between cancer cell fatty acid metabolism and oncogenic signaling as resource for novel treatment strategies against ovarian cancer
}

R. Wagner ${ }^{1}$, D. Veigel ${ }^{1}$, K. Pröstling ${ }^{1}$, G. Stübiger ${ }^{2}$, M. Grusch $^{1}$, J. Weghuber ${ }^{3}$, C. Singer ${ }^{4}$, H. Karlic ${ }^{5}$, R. Colomer $^{6}$, M.L. López-Rodríguez ${ }^{7}$, B. Benhamú ${ }^{7}$, F.G. Hegardt ${ }^{8}$, D. Serra ${ }^{8}$, J. García $^{9}$, P. Valent ${ }^{10}$, T.W. Grunt ${ }^{10}$

\footnotetext{
${ }^{1}$ Medical University Vienna, Comprehensive Cancer Center, Department of Medicine I, Vienna, Austria;

${ }^{2}$ Medical University Vienna, Comprehensive Cancer Center, Center of Physiology and Pharmacology, Vienna, Austria;

${ }^{3}$ University of Applied Sciences, School of Engineering and Environmental Sciences, Wels, Austria;

${ }^{4}$ Medical University Vienna, Comprehensive Cancer Center, Department of Obstetrics/Gynecology, Vienna, Austria;

${ }^{5}$ Medical University Vienna, Ludwig Boltzmann Cluster Oncology, Vienna, Austria;

${ }^{6}$ Spanish National Cancer Research Center CNIO, Department of Medical Oncology, Madrid, Spain;

${ }^{7}$ Universidad Complutense, Departamento de Química Orgánica I, Madrid, Spain;

${ }^{8}$ Universitat de Barcelona, Department of Biochemistry and Molecular Biology, Barcelona, Spain;

${ }^{9}$ Universitat de Barcelona, Institut de Biomedicina, Barcelona, Spain;

${ }^{10}$ Medical University Vienna, Comprehensive Cancer Center, Department of Medicine I \& Ludwig Boltzmann Cluster Oncology, Vienna, Austria
}

The metabolic oncogene fatty acid synthase (FASN) is overexpressed in $80 \%$ of ovarian cancers $(\mathrm{OC})$ and indicates poor prognosis. Exposure of OC to inhibitors of FASN elicits a complex stress response that interferes with receptor-PI3K-mTORC1 signaling (briefly designated 'PI3K pathway'). Here we demonstrate that FASN inhibitors capitalize on multiple mechanisms to interfere with the PI3K pathway, and that silencing this cascade is crucial for the anticancer action of the drugs. Data were obtained using thin-layer chromatography, ELISA, Western blotting, quantitative micropatterning and growth assays, respectively. Exposure of OC cells (SKOV3, OVCAR-3) to FASN inhibitors (C75, G28UCM) causes lipid redistribution toward storage lipids, whereas membrane lipid rafts and signaling lipids are diminished, which significantly impairs EGF receptor/ErbB/HER function and expression. A severe depletion of phosphatidylinositol $(3,4,5)$ trisphosphate (PIP3), which represents the crucial product of PI3K action, is associated with drug-dependent silencing of AKT. Moreover, FASN blockers rapidly stimulate expression of the stress response gene REDD1 (RTP801/Dig2/DDIT4) followed by slow activation of the energy sensor AMPK. Induction of these mTORC1 upstream repressors has been found to block downstream phosphorylation of ribosomal S6 protein. Moreover, long-term stress imposed by persistent FASN blockade leads to accelerated degradation of signaling proteins. Interestingly, concurrent targeting of the PI3K pathway using the dual PI3K/mTOR blocker NVP-BEZ235 does not aggravate the FASN anticancer drug effects, indicating that $\mathrm{PI} 3 \mathrm{~K}$ is already maximally silenced due to FASN blockade. In contrast, exogenous expression of constitutive active AKT counteracts FASN inhibitor-mediated mTORC1 silencing and abrogates growth arrest. Elimination of $\mathrm{PI}$ KK downstream activity thus appears crucial for the anticancer effect of FASN blockers. On the other hand, silencing PI3K signaling by FASN inhibitors was found to release a negative feedback loop toward MAPK. Thus, FASN drug-mediated PI3K silencing is associated with cross-activation of ERK1/2. Accordingly, co-treatment with the MEK inhibitor selumetinib (AZD6244) significantly improves the anticancer action of FASN inhibitors, whereas introduction of constitutive active MEK does not alter FASN drug-induced growth inhibition. Collectively these data demonstrate that FASN inhibitors utilize a whole panel of different mechanisms to abrogate receptor-PI3K-mTORC1 signaling, which represents at least one of the crucial mechanisms of anticancer action of FASN targeting drugs. Supported by Medical Scientific Fund of the Mayor of the City of Vienna \& 'Initiative Krebsforschung' of the Medical University Vienna, Austria.

\section{No conflict of interest.}

